\title{
Serratus Anterior Plane Block as a Regional Analgesia Technique for Post Thoracotomy and Thoracoscopy Pain: Review Article
}

\author{
Fawzy Abbas Badawy, Abd El-Rahman Hasan Abd El-Rahman, Salah Ahmed Mohamed, Asmaa Saad-Eldeen \\ Farghaly Gad-Allah*, Omima Emad Eldin Mohamed, Khaled Abdelfattah Mohamed Abdelfattah \\ Department of Anaesthesiology, ICU and Pain Management, Faculty of Medicine, Sohag University, Egypt. \\ *Corresponding author: Asmaa Saad-Eldeen Farghaly Gad-Allah, Mobile: (+20)01020283823, \\ E-mail: asmaasaad@med.sohag.edu.eg
}

\begin{abstract}
Background: Chest wall surgeries are accompanied by severe postoperative pain. Inadequate relief of this pain may lead to both pulmonary complications as lung atelectasis and infection and chronic post thoracotomy pain syndrome. Regional analgesic modalities are important portion of the multimodal therapeutic approach suggested for the management of post thoracotomy pain.

Objective: To evaluate serratus anterior plane block as a regional analgesia technique for post thoracotomy and thoracoscopy pain.

Recent Findings: Serratus anterior plane block (SAPB), a regional analgesic modality developed by Blanco et al. in 2013, has shown good analgesic effect after thoracotomy and thoracoscopy in many case reports and clinical trials. In such block, a local anesthetic is injected in the fascial plane deep or superficial to the serratus anterior muscle leading to block of lateral cutaneous branches of the intercostal nerves. This provides a sensory block of T2-T9 dermatomes.

Conclusion: Serratus anterior plane block as a fascial plane block can be a preferred regional analgesia technique for both post-operative pain management with procedures involving anterolateral chest wall as thoracotomy, thoracoscopy and breast surgery and in cases of multiple rib fractures. This is owing to its easy technique, effective pain relief and potentially better side effects profile compared to other regional modalities and systemic opioids.
\end{abstract}

Keywords: Serratus anterior plane block, Post thoracotomy pain.

\section{INTRODUCTION}

Thoracotomy and thoracoscopy are associated with acute severe pain, inadequate relief of which will immediately affect pulmonary function and patient comfort. In addition, it will lead to longer term pain (post thoracotomy pain syndrome) that interferes with patient's returning to his regular activities for extended time $(\mathbf{1}, \mathbf{2})$. Pleural irritation, costovertebral joints dislocation, intercostal nerves injury and retraction and resection or fracture of ribs are involved factors in evolving such severe pain ${ }^{(3)}$. Providing analgesia for thoracic surgery can be challenging for a variety of reasons as thoracic surgery patients are often elderly and/or frail with multiple comorbidities ${ }^{(\mathbf{1})}$.

For thoracic surgery, enhanced recovery after surgery (ERAS) guidelines (4) advised a multimodal approach for post thoracotomy acute pain management including:

$>$ Cognitive behavioral modalities to relief anxiety as guided imagery, intraoperative positive suggestions, limiting noise and playing music during anesthetic induction and other relaxation techniques can reduce anxiety. Patient education and providing proper information also have important rule in experiencing less pain ${ }^{(5)}$.

$>$ Multimodal systemic analgesia by using synergistic effects of different analgesic types aiming to avoid or minimize opioids use to avoid opioids related side effects that may interfere with achieving ERAS targets like postoperative nausea/vomiting control, quick resuming of oral diet and early mobilization. These include acetaminophen, Non-steroidal antiinflammatory drugs, N-methyl-D-aspartate (NMDA) antagonists, Gabapentin, Glucocorticoids and Opioids (4).

Intraoperative regional analgesia: Thoracic epidural analgesia was considered by many studies as the gold standard, as it was more effective when compared to many other regional analgesic modalities used for post thoracotomy pain treatment. Though, associated risks with the use of epidural analgesia are becoming more obvious than previously thought, including hypotension, urinary retention, technique failure, muscle weakness and other epidural related complications ${ }^{(6,7)}$. Thoracic paravertebral block analgesia can be used as a unilateral block of both somatic and sympathetic nerves and is suitable in unilateral thoracic procedures with equivalent analgesic effect, better side effects profile and probably less respiratory complications compared to thoracic epidural analgesia $(\mathbf{6}, \mathbf{8})$. Other regional analgesia techniques were tried aiming easier access and fewer complications, like intercostal nerve blocks and intrapleural block and showed better analgesic effect than placebo ${ }^{(6,9,10)}$, while others like erector spinae plane block still evolving ${ }^{(11)}$. In 2013, Blanco et al. ${ }^{(12)}$ presented serratus anterior plane block (SAPB), a new feasible and safer ultrasound guided technique for thoracic wall nerve block, as an alternative to the standard regional analgesic techniques as thoracic epidural and paravertebral block. 
Anatomy: The serratus anterior is located laterally in the thoracic wall and comprises the medial border of the axilla region (Figure 1). It consists of several strips that originate from the lateral aspects of the $1^{\text {st }}$ to $8^{\text {th }}$ ribs and attach to the scapula in the medial border of its costal surface. It is innervated by long thoracic nerve (C5, C6, and C7) and its function is to hold the scapula against the chest cage and to move the scapula forward and upward allowing the arm to be raised over 90 degrees ${ }^{(13,14)}$. Potential spaces exist both deep to the serratus anterior muscle between it and chest wall, and superficial to it underneath the latissimus dorsi muscle, which lies superficial to the serratus anterior.

The intercostal nerves give their lateral cutaneous branch near the angle of its related ribs. Piercing the intercostal muscles, the lateral cutaneous branches intercostal nerves divide to give anterior and posterior branches (Figure 2). Both branches pierce the serratus anterior muscle and supply the skin covering the pectoralis major, scapula and latissimus dorsi. Therefore, the thoracic intercostal nerves are blocked when a local anesthetic is injected in the potential space either deep or superficial to the serratus muscle, providing analgesia to the anterolateral chest wall extended from $\mathrm{T} 2$ to $\mathrm{T} 9(\mathbf{1 2}, \mathbf{1 3}, \mathbf{1 4})$.

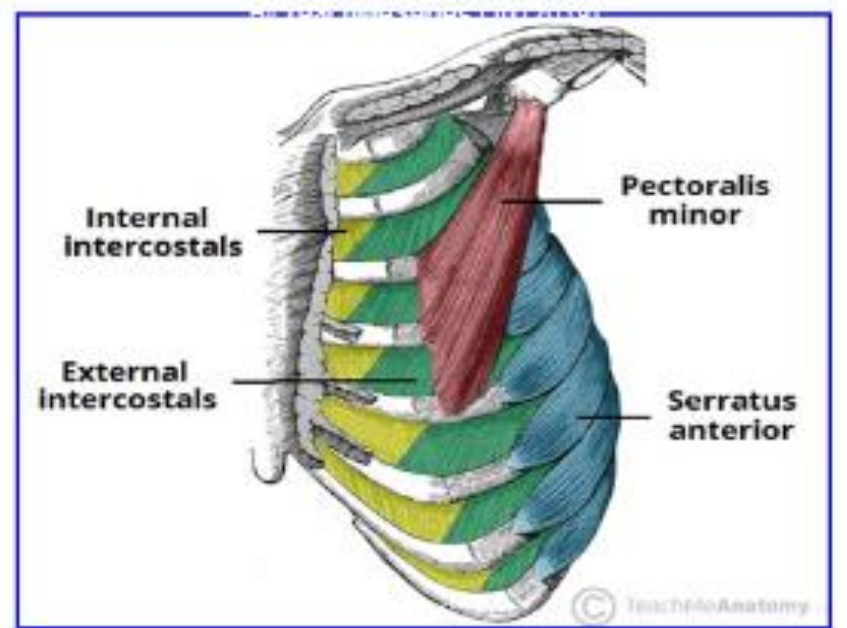

Figure (1): The internal and external intercostals of the thoracic wall ${ }^{(\mathbf{1 4})}$

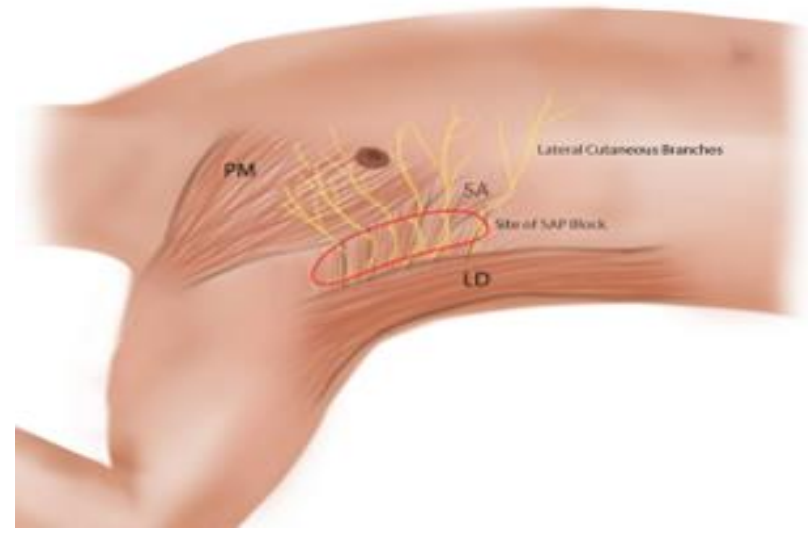

Figure (2): A diagram showing applied anatomy for serratus anterior plane block. SA: serratus anterior, PM: pectoralis major and LD: latissimus dorsi ${ }^{(\mathbf{1 5})}$

Sonoanatomy:
Chest wall is composed of four major components (Figure 3): the skin that forms a thin hyperechoic outer layer $(1-3 \mathrm{~mm})$, subcutaneous fat layer of varying thickness that is hypoechoic and lobulated and a complex variety of muscles. All the three layers are supported by cartilages and bones (the ribs, clavicles, sternum and the sternoclavicular joint). Only the anterior face of bony parts of the ribs is visible on ultrasound as it completely attenuates the spread of the ultrasound waves ${ }^{(16)}$.

The muscles of the chest wall on ultrasound have the characteristic appearance of striated muscles as parallel hypoechoic fibers separated by fine echogenic bands. The superficial muscles of the thoracic wall include: the pectoralis major and minor, the serratus anterior and the subclavius, while the deep muscles layer is formed of the external and internal intercostal muscles. The innermost intercostal and the transversus thoracis muscles cannot be seen by ultrasound. More dorsally, a superficial group of muscles including the latissimus dorsi, the trapezius and serratus posterior can be identified ${ }^{(16)}$.

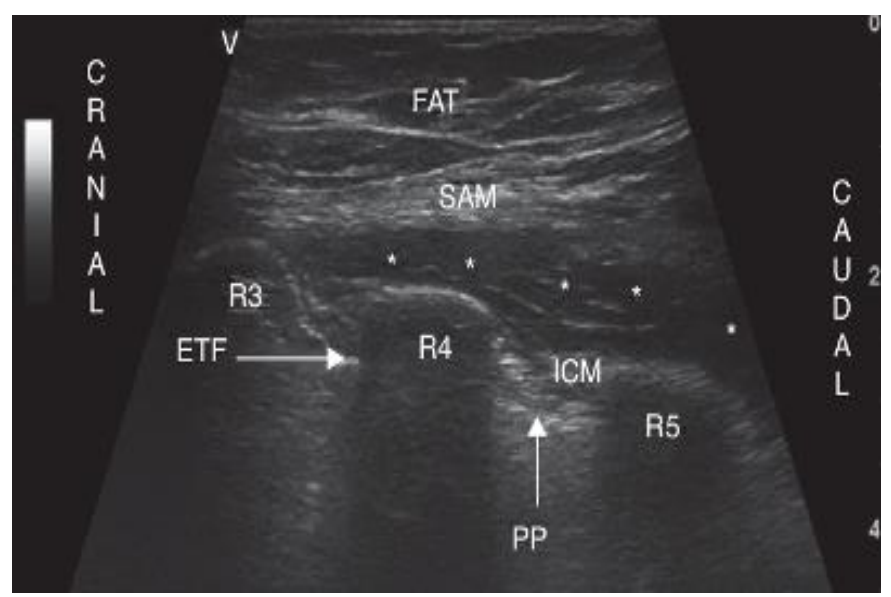

Figure (3): Ultrasound of the thoracic wall. ICM: intercostal muscles; SAM: serratus anterior muscle; R3: third rib; R4: fourth rib; R5: fifth rib; ETF: enthoracic fascia; PP: parietal pleura; FAT: subcutaneous fat; * indicates hydro dissection of the fascial plane with local anesthetic solution in this space ${ }^{(\mathbf{1 6})}$.

\section{Rationale:}

The increasing usage of ultrasonography in identifying tissue layers, particularly fascial layers, led to the evolving of many newer interfascial analgesia injection techniques for both chest and abdominal wall. An example is the SAPB, which can be considered as a modification of Pecs II block. In Pecs II block, local anesthetic is injected into two fascial spaces by dividing the dose between the pectoral fascia between the pectoralis major and pectoralis minor muscles and the fascial space under the pectoralis minor muscle between the clavipectoral fascia and the superficial border of the serratus anterior muscle ${ }^{(17)}$. In SAPB, the procedure is performed in the axillary region more lateral and posterior to the location in which Pecs II block is performed, where lateral cutaneous branches of 
(T3-T9) intercostal nerves, intercostobrachial nerve, thoracodorsal nerve, and long thoracic nerve are located in the fascial compartment between the serratus anterior muscle and the latissimus dorsi muscle, in the area between the mid and posterior axillary lines ${ }^{(18)}$. To provide analgesia of the lateral thoracic wall, this block is targeting primarily the thoracic intercostal nerves by approaching one of two fascial spaces either superficial to the serratus anterior muscle underneath the latissimus dorsi muscle or deep to the serratus anterior muscle between the serratus muscle and the thoracic wall ${ }^{(12,19)}$.

\section{Technique:}

Preparation: Informed consent, aseptic precautions, standard noninvasive monitoring $(\mathbf{1 2 , 2 0 )}$.

Probe: Linear ultrasound transducer (10-12 $\mathrm{MHz}$ ) is usually used (12, 20, and 21). Linear ultrasound transducers with variable frequency ranges were also used ${ }^{(22,23,24,25,26, \text { and 27). }}$.

Patient position: The procedure can be performed in one of two patient positions:

1) While the patient is in the supine position (Figure 4) with the ipsilateral arm abducted to $90^{\circ}$. This position can be used in poly traumatized patients as in case of multiple rib fractures $(\mathbf{1 2 , 2 2 , 2 7}$, and 28).

2) While the patient is in a lateral position (Figure 5) with the diseased side up. This position can be used in patients undergoing thoracotomy or thoracoscopy after anesthesia induction ${ }^{(20,21,23,}$ $24,25,26$, and 29)

\section{Probe position and orientation (Figures 4, 5):}

The probe is positioned over the midclavicular area of the chest cage in a sagittal plane. Then ribs are counted laterally and inferiorly, until the fifth rib is recognized in the midaxillary line. The latissimus dorsi (posterior and superficial), teres major (superior) and serratus muscles (inferior and deep) are then easily distinguishable by ultrasound covering the fifth rib. Thoracodorsal artery can be used as a further reference point. This aids in the identification of the plane superficial to the serratus muscle ${ }^{(12,24, \text { and } 27)}$. In other studies, SABP was done while the probe is placed in different orientation being parallel to and between the 5 th and 6th ribs in the mid-axillary region ${ }^{(20,21)}$.

Needle insertion: G-22 $50 \mathrm{~mm}$ needle is commonly used (12, 20). Variable needle gauge and length were used varying from G-18 to G-25 and 50 $\mathrm{mm}$. to $100 \mathrm{~mm}^{(21,22,24,25,26,27, \text { and } 29)}$. The needle depth required to reach the identified region is around one to two centimeters ${ }^{(\mathbf{1 2})}$.

The needle is introduced in plane keeping respect to the ultrasound probe from superoanterior to posteroinferior. After confirming negative aspiration, correct placement of the needle tip is ensured by injecting $3 \mathrm{ml}$ normal saline to hydro dissect the targeted plane ${ }^{(21,22,25, \text { and } 29)}$.

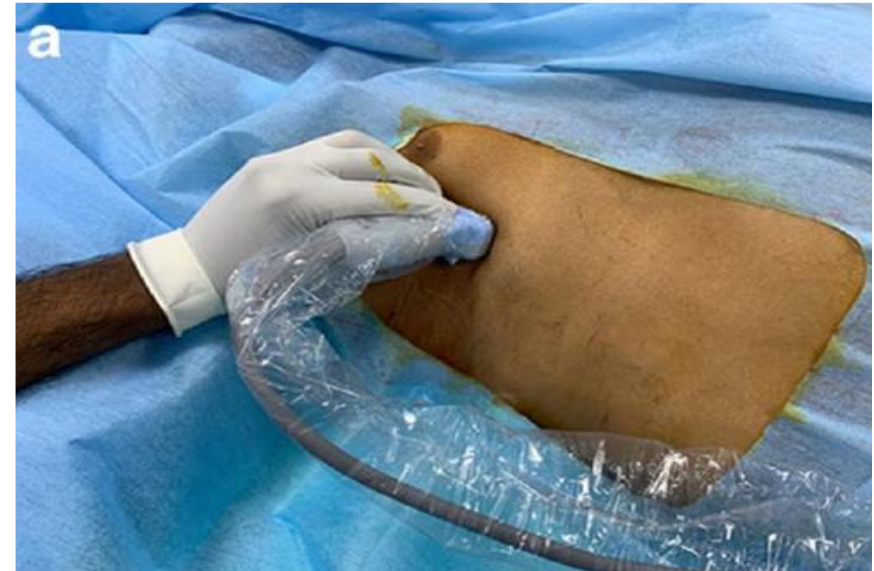

Figure (4): Patient in supine position ${ }^{(15)}$.

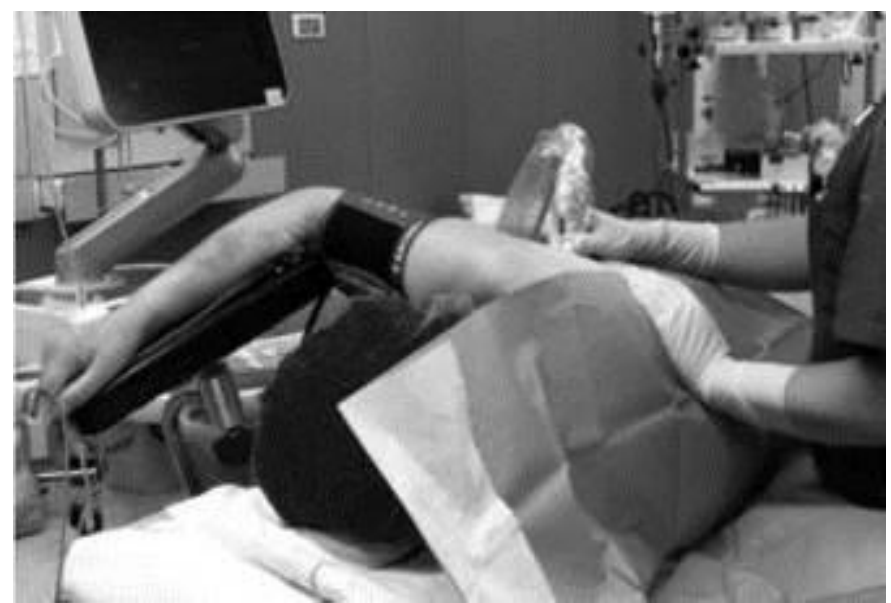

Figure (5): Patient in lateral position ${ }^{(20)}$.

Local anesthetic injection: A local anesthetic is then injected under continuous ultrasound guidance either superficial (Figures 6, 7) ${ }^{(12,20,21,23,25,24,26,29, \text { and 30) }}$ or

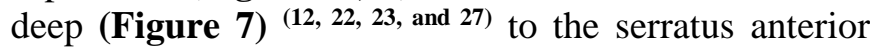
muscle confirming good spread between latissimus dorsi and the serratus or deep to serratus muscle. Superficial approach is preferred and may give a wider area (a more posterior spread) of analgesia and a greater duration of action in spite of lacking evidence to support either choice ${ }^{(\mathbf{1 2})}$.

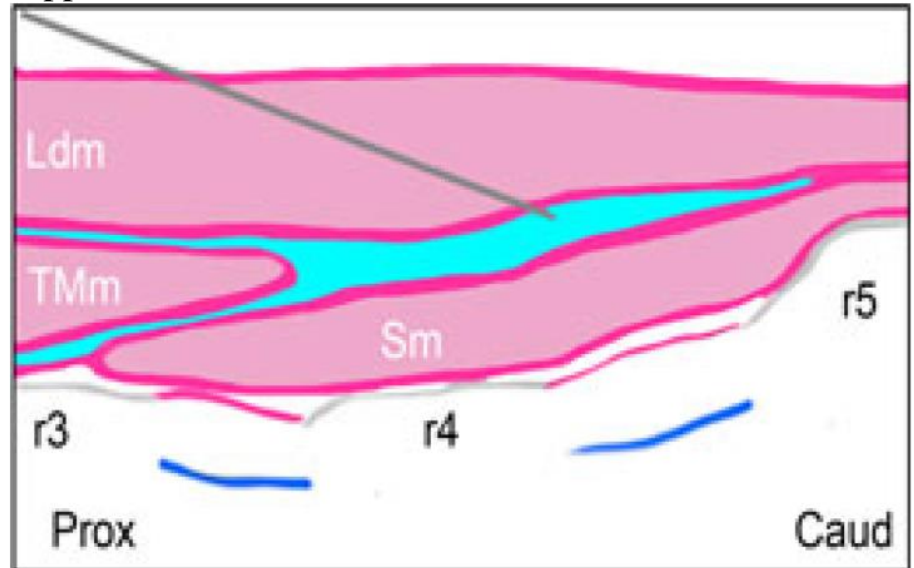

Figure (6): A graph representing the distribution of local anaesthetic (in blue) during serratus plane block. Ldm: latissimus dorsi; Tmm: Teres major; Sm: serratus muscle; the ribs: three (r3), four (r4) and rib five (r5); Prox: proximal and Caud: caudal ${ }^{(\mathbf{1 2})}$. 

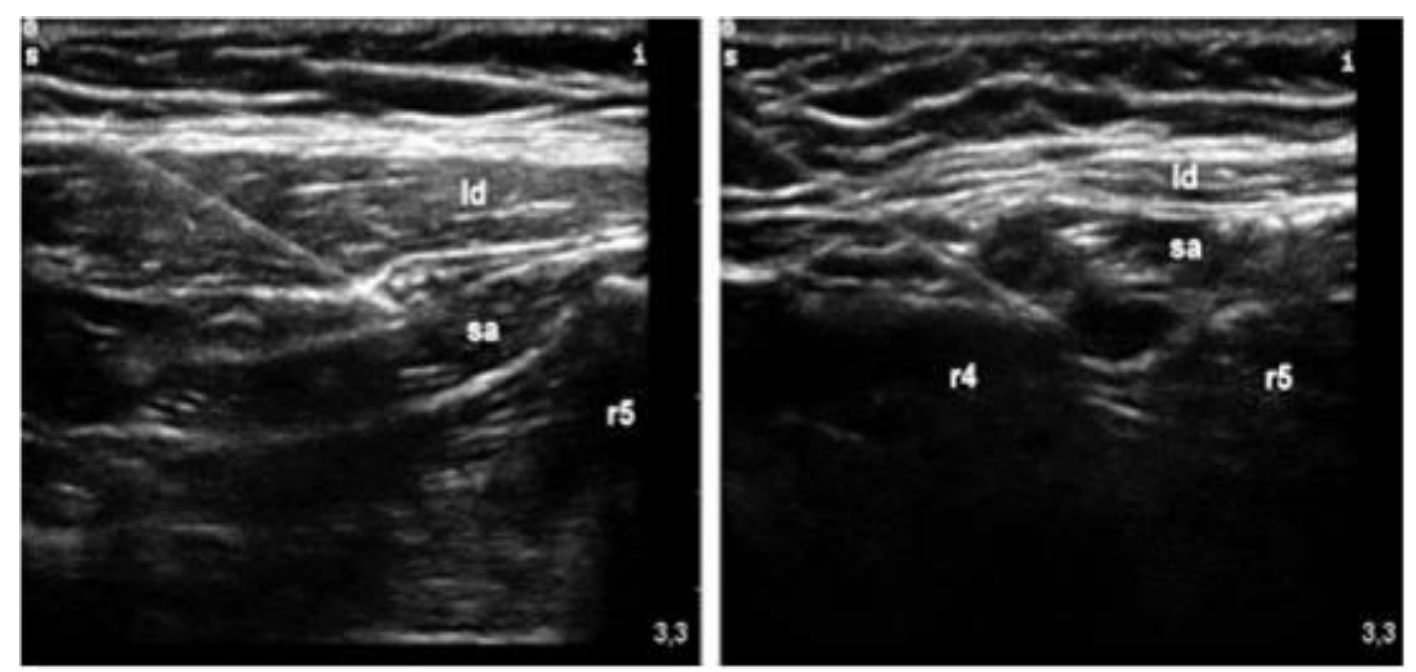

Figure (7): Sonographic images showing the fascial planes above (left) and below (right) the serratus anterior muscle is dissected during local anesthetic injection. Ld: latissimus dorsi; Sa, serratus anterior; r4: fourth rib and r5: fifth rib (12)

Catheter insertion and needle removal: After injection, a catheter can be threaded into the fascial plane via the needle before its removal and secured to allow infusion of local anesthetic to give prolonged analgesia ${ }^{(20,22)}$. If single injection approach is planned, the needle is removed immediately after local anesthetic injection without catheter threading $(\mathbf{1 2}, \mathbf{2 1}, \mathbf{2 3}$, $24,25,26,27,29$, and 30 )

Local anesthetic agent: The local anesthetic used varied among studies in type, dose and concentration. Ropivacaine was used in concentrations of $0.25,0.375$ or $0.5 \%$ either in a fixed dose $20: 30 \mathrm{~mL}$ or based on body weight $0.4 \mathrm{~mL} / \mathrm{Kg}$. In case of subsequent infusion, lower concentrations are used

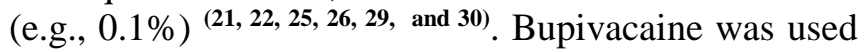
in concentrations 0.25 or $0.5 \%$ either in a fixed dose 20:30 $\mathrm{mL}^{(24,27)}$ or based on body weight $2 \mathrm{mg} / \mathrm{Kg}{ }^{(23)}$. Levobupivacaine $30 \mathrm{~mL}$ was used in concentrations $0.25 \%$ followed by $5 \mathrm{~mL} /$ hour infusion of $0.125 \%$ concentration ${ }^{(20)}$.

Additives to local anesthetics: In one study, dexamethasone $8 \mathrm{mg}$ was added to the injected local anesthetic bolus $(0.25 \%$ bupivacaine $){ }^{(23)}$. While in another study, fentanyl 1 microgram $/ \mathrm{mL}$ was added to the local anesthetic (ropivacaine $0.1 \%$ ) infused via the fixed catheter but not with initial bolus ${ }^{(22)}$.

\section{Indications:}

SAPB is designed primarily to block the lateral cutaneous branches of thoracic intercostal nerves to provide analgesia of the lateral chest wall ${ }^{(\mathbf{1 2})}$. Many studies and case reports described its use in

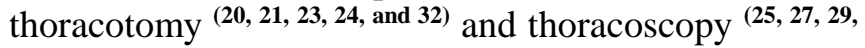
and 30) procedures. Additionally, it is used in surgery in the region of the breast and axilla, like in modified radical mastectomy ${ }^{(26,31)}$ and unilateral multiple rib fractures ${ }^{(22,28)}$.

\section{Contraindications:}

There are very few contraindications for SAPB. Absolute contraindications include patient' refusal (informed consent should be obtained from patient preoperative), allergy to local anesthetics and local infection at the needle insertion site. Relative contraindications include situations that make anatomical landmarks difficult to be identified by ultrasound, for example morbid obesity or distorted anatomy as in case of surgical emphysema, intercostal drain placement or previous surgery at the insertion site $^{(19,33)}$

\section{Advantages:}

SAPB has many advantages. It is technically easy as it is a superficial block. It also can be performed with patients either in lateral or supine position, therefore, it is particularly beneficial in trauma patients as other concurrent injuries may prevent patients from sitting or rolling laterally to perform either paravertebral block or thoracic epidural. In addition, it is appropriate for patients who have associated head injuries or spinal trauma where epidural and paravertebral blocks are contraindicated. Besides, it can be used in thrombolysed or anticoagulated patients. If a catheter is inserted and secured for local anesthetic infusion, patients can move safely with catheter in situ ${ }^{(\mathbf{1 9}, \mathbf{3 3})}$.

\section{Complications:}

The procedure is generally easy and safe with better side effects profile compared to other regional modalities and systemic opioids. Complications are unlikely with skilled hands. It includes pneumothorax, nerve injury, vascular puncture, technique failure leading to inadequate block, infection (prevented by proper antiseptic preparation) and allergy to local anesthetic (urticaria, bronchospasm or hypotension may rarely occur) $(\mathbf{1 9}, 33,34)$. Local anesthetic systemic toxicity is rare with correct administration. Excessive plasma levels may happen due to overdose, unintentional IV injections or slow metabolic degradation resulting mainly in CNS (up to generalized tonic clonic convulsions and respiratory arrest) and 
cardiovascular effects. Bupivacaine is obviously cardiotoxic (highly arrhythmogenic) compared to other local anesthetics $(\mathbf{1 9}, \mathbf{3 4 , 3 5 )}$.

\section{CONCLUSION}

Serratus anterior plane block as a fascial plane block that can be a preferred regional analgesia technique for both post-operative pain management with procedures involving anterolateral chest wall as thoracotomy, thoracoscopy and breast surgery and in case of multiple rib fractures. This is owing to its easy technique, effective pain relief and potentially better side effects profile compared to other regional modalities and systemic opioids.

Conflicts of interest: There are no conflicts of interest.

\section{REFERENCES}

1. Gottschalk A, Cohen S, Yang $S$ et al. (2006): Preventing and treating pain after thoracic surgery. Anesthesiology, 104: 594-600.

2. Ochroch E, Gottschalk A, Augostides J et al. (2002): Long term pain and activity during recovery from major thoracotomy using thoracic epidural analgesia. Anesthesiology, 97: 1234-44.

3. Ochroch E, Gottschalk A (2005): Impact of acute pain and its management for thoracic surgical patients. Thorac Surg Clin., 15: 105-21.

4. Batchelor T, Rasburn N, Abdelnour-Berchtold E et al. (2019): Guidelines for enhanced recovery after lung surgery: recommendations of the Enhanced Recovery After Surgery $\left(\right.$ ERAS $\left.^{\circledR}\right)$ Society and the European Society of Thoracic Surgeons (ESTS). European Journal of Cardio-Thoracic Surgery, 55 (1): 91-115.

5. Chou $\mathrm{R}$, Gordon $\mathrm{D}$, de Leon-Casasola $\mathrm{O}$ et al. (2016): Management of Postoperative Pain: a clinical practice guideline from the American pain society, the American Society of Regional Anesthesia and Pain Medicine, and the American Society of Anesthesiologists' committee on regional anesthesia, executive committee, and administrative council. The Journal of Pain, 17: 131-57.

6. Joshi G, Bonnet F, Shah R et al. (2008): A systematic review of randomized trials evaluating regional techniques for post thoracotomy analgesia. Anesth Analg., 107: 1026-40.

7. Cook T, Counsell D, Wildsmith J (2009): Royal College of Anaesthetists Third National Audit Project . Major complications of central neuraxial block: report on the Third National Audit Project of the Royal College of Anaesthetists. Br J Anaesth., 102: 179-90.

8. Yeung J, Gates S, Naidu B et al. (2016): Paravertebral block versus thoracic epidural for patients undergoing thoracotomy. Cochrane Database Syst Rev., 2: 9121-24.

9. D'Andrilli A, Ibrahim M, Ciccone A et al. (2006): Intrapleural intercostal nerve block associated with mini-thoracotomy improves pain control after major lung resection. Eur J Cardiothorac Surg., 29: 790-4.

10. Morris C J, Bunsell R (2014): Intrapleural blocks for chest wall surgery. Anaesthesia, 69: 85-6.

11. Jain K, Jaiswal V, Puri A (2018): Erector spinae plane block: Relatively new block on horizon with a wide spectrum of application - A case series. Indian J Anaesth., 62(10):809-813.

12. Blanco R, Parras T, McDonnell J et al. (2013): Serratus plane block: a novel Ultrasound guided thoracic wall nerve block. Anaesthesia., 68: 1107-13.

13. Jhonson D (2005): Chest Wall. In: Shah D, editors. Gray's Anatomy. Elsevier Churchill Livingstone, Pp: 951-968.

14. Jones O (2019): The Muscles of the Thoracic Cage. https://teachmeanatomy.info/ thorax /muscles/thoraciccage

15. Gautam S, Agarwal A (2019): Serratus Plane Block in the Management of Post-operative Thoracotomy Pain. Curr Anesthesiol Rep., 9: 459-463.

16. Lee R, Griffith J, Ng A et al. (2015): Sonography of the chest wall: A pictorial essay. J Clin Ultrasound, 43: 525-537.

17. Blanco R, Fajardo M, Parras Maldonado T (2012): Ultrasound description of Pecs II (modified Pecs I): a novel approach to breast surgery. Rev Esp Anestesiol Reanim., 59: 470-475.

18. Porzionato A, Macchi V, Stecco C et al. (2012): Surgical anatomy of the pectoral nerves and the pectoral musculature. Clinical Anatomy, 25: 559-75.

19. Blanco R, Barrington $M$ (2017): Pectoralis and serratus plane blocks. In: Rafael Blanco R, Barrington MJ, eds. Textbook of Regional Anesthesia and Acute Pain Management. 2nd ed. New York, NY: McGrawHill Publishing. https://accessanesthesiology. mhmedical.com/content.aspx?bookid=2070\&sectionid= 157602747

20. Khalil A, Abdallah N, Bashandy G et al. (2017): Ultrasound guided Serratus Anterior Plane Block versus Thoracic Epidural Analgesia for Thoracotomy Pain. Journal of Cardiothoracic and Vascular Anesthesia., 31: $152-158$.

21. Chen G, Li1 Y, Zhang Y et al. (2019): Effects of serratus anterior plane block for postoperative analgesia after thoracoscopic surgery compared with local anesthetic infiltration: a randomized clinical trial. Journal of Pain Research, 12: 2411-2417.

22. Jadon A, Jain P (2017): Serratus Anterior Plane BlockAn Analgesic Technique for Multiple Rib Fractures: A Case Series. American J Anesth Clin Res., 3 (1): 1-4.

23. Semyonov M, Fedorina E, Grinshpun J et al. (2019): Ultrasound guided serratus anterior plane block for analgesia after thoracic surgery. Journal of Pain Research, 12: 953-960.

24. Saad F, El Baradie S, Aliem M et al. (2018): Ultrasound guided serratus anterior plane block versus thoracic paravertebral block for perioperative analgesia in thoracotomy. Saudi Journal of Anaesthesia., 12 (4): 565-75.

25. Kim D, Oh Y, Lee J et al. (2018): Efficacy of Ultrasound guided serratus plane block on postoperative quality of recovery and analgesia after video assisted thoracic surgery: a randomized, triple-blind, placebocontrolled study. Anesthesia \& Analgesia, 126 (4): 1353-1361.

26. Yao Y, Li J, Hu H et al. (2019): Ultrasound guided serratus plane block enhances pain relief and quality of recovery after breast cancer surgery: a randomised controlled trial. European Journal of Anaesthesiology (EJA), 36 (6): 436-441. 
27. Ökmen K, Ökmen B (2018): Evaluation of the effect of serratus anterior plane block for pain treatment after video assisted thoracoscopic surgery. Anaesthesia Critical Care \& Pain Medicine, 37 (4): 349-353.

28. Durant E, Dixon B, Luftig et al. (2017): Ultrasoundguided serratus plane block for ED rib fracture pain control. The American Journal of Emergency Medicine, 35: 197-203.

29. Lee J, Kim S (2019): The effects of Ultrasound guided serratus plane block, in combination with general anesthesia, on intraoperative opioid consumption, emergence time, and hemodynamic stability during video assisted thoracoscopic lobectomy: a randomized prospective study. Medicine, 98 (18): 155-161.

30. Wang $L$, Wang $Y$, Zhang $X$ et al. (2019): Serratus anterior plane block or thoracic paravertebral block for postoperative pain treatment after uniportal video assisted thoracoscopic surgery: a retrospective propensity-matched study. Journal of Pain Research, 12: 2231-36.
31. Datu M, Prasetyadhi J (2020): Serratus anterior plane block in modified radical mastectomy surgery: a case series. JA Clin Rep., 6: 82.

32. Ökmen K, Ökmen B (2017): The efficacy of serratus anterior plane block in analgesia for thoracotomy: a retrospective study. Journal of Anesthesia, 31 (4): 579585.

33. May L, Hillermann C, Patil S (2016): Rib fracture management. BJA Education, 16 (1): 26-32.

34. Brunton L, Chabner B, Knollman B (2010): Goodman and Gilman's. The Pharmacological Basis of Therapeutics, Twelfth Edition. San Diego, California, 2010.

https://accessmedicine.mhmedical.com/content.aspx?bo okid=1613\&sectionid $=102124003$

35. Picard J, Meek T (2006): Lipid emulsion to treat overdose of local anaesthetic: the gift of the glob. Anaesthesia, $61 \quad$ (2): $107-9$. 